\title{
Enjeux socio-politiques dans la mise en œuvre de l'apprentissage par problèmes (APP)
}

\section{Mamadou Bhoye BAH}

\section{(2) OpenEdition \\ 12 Journals}

Édition électronique

URL : http://journals.openedition.org/ere/782

DOI : 10.4000/ere.782

ISSN : 2561-2271

Éditeur

Centr'ERE

\section{Édition imprimée}

Date de publication : 20 décembre 2016

ISSN : 1373-9689

\section{Référence électronique}

Mamadou Bhoye BAH, « Enjeux socio-politiques dans la mise en œuvre de l'apprentissage par problèmes (APP) », Éducation relative à l'environnement [En ligne], Volume 13 - 2 | 2016, mis en ligne le 20 décembre 2016, consulté le 21 février 2020. URL : http://journals.openedition.org/ere/782 ; DOI : $10.4000 /$ ere. 782 


\title{
Enjeux socio-politiques dans la mise en œuvre de l'apprentissage par problèmes (APP)
}

\author{
Mamadou Bhoye BAH
}

1 Cet article se penche sur les enjeux socio-politiques tels que perçus par vingt et un étudiants du programme de Master en sciences de l'environnement du Centre d'étude et de recherche en environnement (CERE), de l'Université Gamal Abdel Nasser de Conakry dans le cadre d'un cours d'éducation relative à l'environnement (ERE). Échelonnée sur cinq mois (de janvier à mai 2011), la mise en œuvre d'une nouvelle approche pédagogique, en l'occurrence l'apprentissage par problèmes (APP), visait le développement de compétences associées au champ de l'ERE chez ces étudiants. La démarche privilégiée est celle d'une étude de cas interprétative et critique. Deux principales stratégies de collecte de données ont été utilisées: l'observation participante et l'entrevue semi-dirigée. Pour situer ce travail, le cadre théorique s'articule autour de trois éléments : l'APP réflexif comme stratégie d'accompagnement des étudiants, les théories sociocognitives de l'apprentissage contextualisé guidant les stratégies d'enseignement-apprentissage et les enjeux socio-politiques. Le déroulement du scénario pédagogique, les modes de collecte de données ainsi que les modalités d'analyse sont exposés dans les choix méthodologiques. Par la suite, les enjeux sociopolitiques de la dynamique de mise en œuvre de l'APP sont présentés et discutés afin d'en dégager des avenues à explorer pour le développement de compétences associées au champ de l'ERE chez ces étudiants.

\section{Mise en contexte}

2 La dégradation de la situation socio-économique de la Guinée a entraîné des conséquences néfastes sur l'accès aux services de base : eau potable, assainissement, soins de santé primaires, accès à l'électricité, etc. Selon le bureau de la Commission européenne en Guinée (2007), les pratiques dans l'industrie minière engendrent des 
impacts négatifs sur l'environnement, la santé des populations locales et des inégalités sociales. En effet, bien que cette industrie génère des emplois à court terme, ceux-ci sont peu nombreux et souvent comblés par les gens de la ville ou d'autres pays, les populations locales n'ayant pas les compétences requises. Par ailleurs, Lamine Barry (2001, p. 3) souligne que le faible niveau d'instruction et la pauvreté de la majorité de la population guinéenne, couplé à un usage inadéquat de ses "abondantes ressources naturelles ", entraine une dégradation de l'environnement au pays. Il serait important de mentionner qu'en Guinée, comme à bien des endroits dans le monde, pour des raisons structurelles, de l'ordre de la macroéconomie, du système de redevance, des choix politiques et de la corruption contribuent fortement à cette dégradation.

Pour ce qui est du secteur de l'éducation, un rapport de la Banque mondiale (2003, p. 8-9) indiquait des taux de diplomation d'études supérieures assez bas (43\%) et des taux de redoublement ( $30 \%$ des étudiants du supérieur par an) parmi les plus élevés de la sous-région ouest africaine. Et pour cause, note Abdoulaye Barry (1998, p. 13), les bases de la scolarisation obligatoire ont été mises en place sans politique appropriée. Cela amène à se questionner non seulement sur la validité des contenus et des stratégies d'enseignement-apprentissage, mais aussi sur la pertinence des apprentissages réalisés.

4 Par ailleurs, les efforts d'amélioration de la qualité de la formation, notamment par le développement des pédagogies actives, restent faibles en milieu universitaire guinéen. L'éducation relative à l'environnement peut trop facilement être considérée de manière réductive comme un simple outil de résolution de problèmes environnementaux. Tenant compte de la tradition éducative guinéenne, de nature transmissive, dans laquelle les étudiants guinéens n'ont pas l'habitude de prendre en charge leur propre apprentissage, le recours à l'APP, pourrait contribuer au renouveau pédagogique universitaire. D'où l'intérêt de cette recherche qui vise à explorer les enjeux socio-politiques associés à la mise en œuvre de cette approche.

\section{Apprentissage par problèmes de type réflexif comme stratégie d'accompagnement}

5 Saint-Jean (1994) identifie deux façons de concevoir l'APP : l'approche curriculaire qui s'inscrit dans un programme (Walton, Matthews, 1989; Boud et Feletti, 1991; Margetson, 1991 ; Albanese et Mitchell, 1993) et l'APP comme stratégie d'enseignement (Walton et Matthews, 1989). Ici, l'APP sera vue comme une stratégie d'accompagnement des étudiants du CERE dans le cadre d'un cours d'ERE. Les séquences de ce cours donnent généralement lieu à un scénario pédagogique organisé autour de trois situations-problèmes à facettes multiples.

En APP, un problème peut être défini comme un ensemble de circonstances dans un contexte donné, nouveau pour l'étudiant, où la seule utilisation des schémas connus ne suffit pas, mais où des nouveaux éléments précis de connaissance et de compréhension doivent être utilisés... (Walton et Matthews, 1989, p. 543)

Dans un tel contexte, l'étudiant est invité à formuler des hypothèses, à examiner une panoplie de solutions possibles et à choisir la plus appropriée (Saint-Jean, 1994). De même, l'idée de s'appuyer sur une approche par compétences favoriserait le recours à des situations contextualisées, ouvertes et intégratives facilitant ainsi une interaction réciproque entre les ressources mobilisées dans les stratégies d'enseignement- 
apprentissage utilisées. Ces attentes et préoccupations trouvent leurs fondements dans des théories contemporaines de l'éducation. (Bertrand, 1998, p. 149)

\section{Théories socio-cognitives de l'apprentissage contextualisé}

7 Pour les besoins de cette recherche, ce sont les théories de l'apprentissage contextualisé (théories sociocognitives de l'éducation) défendues par des chercheurs rattachés à l'Institute for Research on Learning en Californie qui sont retenues (Greeno, 1988a et b; Brown, Collins et Duguid, 1989). Notre analyse prend appui sur les travaux de Lave et de Bertrand selon qui « nous ne pouvons séparer l'acquisition de connaissances de son contexte pédagogique, culturel et social » (1988, p. 149). Ces auteurs s'accordent pour soutenir que les théories de l'apprentissage contextualisé reposent sur un principe, à savoir que la connaissance dépend essentiellement de l'action et de la culture.

[...] Les individus traitent, représentent et rappellent leurs connaissances dans leurs relations les uns avec les autres et en fonction du milieu social où ils se trouvent. Par conséquent, nous devrions étendre le champ de notre théorie intra-individuelle aux activités et aux interactions sociales de tous les jours. (Lave, 1988, p. 149)

Dans le même ordre d'idées, Bertrand (1998) fait observer que l'on doit sortir les connaissances de leur contexte scolaire, les replacer dans leur contexte originel et accompagner les étudiants à l'aide d'activités authentiques. Un apprentissage contextualisé suppose la création de situations où l'étudiant est en interaction dynamique avec son environnement.

Si l'apprentissage est associé à une action de la part de celui qui apprend, il est également associé à une situation ou, si l'on veut, au contexte dans lequel cette connaissance est ancrée. Vouloir dissocier l'apprentissage de son contexte, c'est rendre l'apprentissage stérile, non significatif, donc voué à l'oubli. (Dalceggio, 1991, p. 5)

9 Pour leur part, Sauvé et Orellana (2008) proposent les termes de contextualisation, décontextualisation et recontextualisation, pour décrire la dynamique de toute démarche authentiquement pédagogique. Cela signifie qu'il faut d'abord apprendre et s'engager dans un contexte précis, pour ensuite se distancier de ce contexte, et favoriser l'établissement de liens où les participants trouvent eux-mêmes des situations où ils peuvent retrouver et appliquer ce qu'ils ont appris.

Concrètement donc, nous souhaitons que les enseignants et les formateurs, quel que soit le courant dans lequel ils se situent, procèdent toujours à partir de situations contextualisées, cherchent à analyser ces situations avec les apprenants, pour en découvrir la structure et s'approprier l'outil qui permet d'y faire face, avant d'inviter ceux-ci à se mettre en quête de situations nouvelles où ils puissent utiliser à nouveau l'outil qu'ils maîtrisent maintenant. (Sauvé et Orellana, 2008, p. 166)

$10 \mathrm{Au}$ bilan, les théories de l'apprentissage contextualisé traitent d'éléments fondamentaux de l'apprentissage tels que la recherche du sens, l'acquisition d'habiletés fonctionnelles et le transfert des apprentissages. C'est d'ailleurs ce dont témoigne Tardif (1997) quant à la nécessité de confronter les apprenants à plusieurs contextes afin qu'ils puissent extraire, d'une manière comparative, les conditions nécessaires et suffisantes au transfert de leurs apprentissages. Tous ces éléments pourraient être pris en compte en cernant les enjeux d'ordre socio-politiques tels que perçus par les étudiants durant la mise en œuvre de l'APP au regard du développement de leurs compétences associées au champ de l'ERE. 


\section{Enjeux socio-politiques}

11 Selon Robottom (2003, p. 89), il y a présence d'un enjeu lorsqu'il existe une controverse et que sa résolution est jugée importante par les parties engagées. Une divergence de points de vue est alors exprimée par des gens ayant des valeurs et des intérêts différents. Le fait d'appréhender ou non l'environnement comme un «objet» éminemment politique représente un enjeu majeur car, pour reprendre les propos de Sauvé (2009), l'ERE vise l'apprentissage des processus de gestion des "choses" publiques et de la démocratie. Elle devrait stimuler l'analyse critique des systèmes et des idéologies politiques au regard de la relation à l'environnement. Cette relation ainsi que les enjeux socio-politiques et éducatifs qui en découlent ne peuvent être réduits à une affaire de compromis social historiquement négocié entre certains acteurs sociaux déterminée par un pseudo-consensus planétaire. De tels enjeux trouvent ainsi échos dans la formation universitaire.

Par ailleurs, selon plusieurs chercheurs (Boud et Feletti, 1991; Margetson, 1991), à l'instar d'autres innovations pédagogiques, l'APP a de la difficulté à se tailler une place dans l'enseignement supérieur en particulier parce que cette approche repose sur une épistémologie de la connaissance différente de celle qui est à la base des approches plus traditionnelles. Pour d'autres (Kaufman et Obenshain, 1985 ; Bridges, 1992 ; Saint-Jean, 1994), l'expérience de l'APP dans plusieurs curricula montre que des étudiants peuvent éprouver des difficultés, notamment à cause de l'inversion du rôle d'étudiant passif à celui d'étudiant actif, et du fait de devoir travailler en équipes dans le processus de construction des savoirs.

En outre, le développement de la sociabilité, de l'aptitude à communiquer et de la capacité d'auto-apprentissage n'est pas toujours exploré en formation par les professeurs (Saint-Jean, 1994, p. 12). L'approche APP serait-elle l'idéale ? À l'instar de Dubeau (2001), on peut affirmer qu'il n'y a pas d'approche pédagogique idéale et qu'il incombe à l'enseignant d'identifier les lacunes à chacune d'elles et de créer sa propre façon de faire à travers différentes stratégies à sa disposition. C'est à la lumière de cet éclairage qu'un regard réflexif a porté sur les enjeux socio-politiques perçus par des étudiants du CERE en situation de formation universitaire prenant appui sur l'APP.

\section{Choix méthodologiques}

14 Cette étude de cas interprétative et critique visait ainsi à cerner les enjeux sociopolitiques, tels que perçus par les étudiants du programme de Master au CERE, dans la mise en œuvre de l'APP'. Le tutorat constitue l'essentiel du scénario pédagogique. Il est décrit à travers les principales étapes correspondant à la démarche d'étude des situations-problèmes telle qu'elle s'est déroulée. D'abord, il y a la saisie d'une situation-problème, lorsque l'étudiant s'interroge et prend du recul. Ensuite, on trouve la mobilisation des savoirs nécessaires à la résolution du problème posé lorsque l'étudiant repère, traite et analyse l'information. La prise de position en amont des discussions en équipe amène l'étudiant à se préparer pour argumenter et défendre son point de vue. Lors de la mise en commun, avec l'écoute des autres arguments et points de vue, l'étudiant reconnaît l'importance de la construction collective des savoirs en confrontant ses propres points de vue avec ceux de ses coéquipiers en vue de résoudre le problème. 

comprendre les enjeux socio-politiques liés à l'exercice d'une démocratie participative en contexte guinéen. À titre d'exemple, dans la mise en œuvre du Plan d'action national pour l'environnement (PNAE) depuis 1994, les démarches d'amélioration de l'environnement sont strictement basées sur le recours aux lois et à la législation. C'est possiblement l'une des raisons pour laquelle, malgré toutes les dispositions prises, les défis socio-écologiques guinéens demeurent encore croissants. 


\section{Défis liés à l'adoption d'une dynamique participative}

Certains participants considèrent que l'adoption d'une dynamique participative constitue un enjeu politique majeur. Pour faire face aux défis et difficultés, les équipes de travail ont dû faire preuve de détermination et de souplesse. Nous avons constaté que la discussion se transformait parfois en confrontation, ce qui est de nature à décourager certains participants. Les propos avancés par l'un d'entre eux sont assez évocateurs des malaises éprouvés.

Quand je pense au diagnostic participatif, il peut y avoir, dans un groupe donné, des individus qui ont tendance à monopoliser la parole. Nous avons vécu à peu près la même chose. Parmi nous, il y en a qui parlaient beaucoup empêchant parfois aux autres de s'exprimer même si ces derniers ont de bonnes idées. (S3)

D'autres encore évoquent des difficultés d'adaptation à la nouvelle méthodologie : « $A u$ début, j'avais du mal à m'adapter. Parce que là, il fallait échanger, tomber sur une entente et à partir de là, résoudre le problème. Pour un début, çà m'avait vraiment dérangé » (C1). Pour la majorité des participants, il n'était pas facile de «Réunir tous les membres de l'équipe pour se rencontrer hors classe " (S3). Dans la même veine, un autre affirme : "Je dirai d'abord qu'on n'était pas habitué à l'APP. Ensuite, on avait des difficultés à nous entendre entre coéquipiers sur certaines questions" (R5). "Vous dites que vous abandonnez, je regrette, ce n'est pas normal et vous ne devez pas imposer vos humeurs à vos coéquipiers. » (R5)

Dans certains cas, l'engagement effectif des étudiants est associé à leur motivation : " Très motivant, tu dois fournir ton maximum » (R5). Un autre fait un constat similaire en comparant l'APP à la méthode conventionnelle : «C'est surtout le degré de participation de l'étudiant qui est apprécié ici. C'est différent de la méthode classique et conventionnelle avec laquelle on téléchargeait des choses dans la tête de l'étudiant. »(P2)

D'autres mentionnent l'engagement de l'étudiant dans une démarche de recherche d'information :

"J'ai surtout apprécié le fait d'effectuer des recherches personnelles d'informations » (C1). Un autre participant renforce cette idée : «Étant plus actif dans la recherche d'informations, on retient davantage les nouvelles connaissances. » (M4)

Par ailleurs, certains participants font état de la nécessité d'engagement pour les communautés afin de garantir la durabilité des actions : "L'engagement effectif de toutes les parties prenantes est garant de la durabilité des actions à entreprendre. Aussi, ce n'est qu'à travers des actions concrètes que l'on peut susciter soutien et sympathie de la part des populations et toujours plus de crédibilité auprès des autorités. » (S3)

26 À l'inverse, comme le font remarquer certains participants, le manque d'engagement qui se manifeste par l'absentéisme a été associé à des difficultés dans les relations interpersonnelles, mais également à une tradition éducative qui ne privilégie pas le travail collaboratif de construction de savoirs, qui requiert de la tolérance au stress, de la souplesse, de l'écoute et du respect de l'autre, de l'ouverture d'esprit, etc.

C'est vrai, il y a eu pas mal de choses notamment l'absentéisme, le manque de coopération de la part de certains. J'ai été taxé d'autoritaire parce que j'ai voulu reprendre les choses en main quand j'ai vu que cela ne marchait pas. C'est pourquoi je me suis rétracté au PROSIT 2. Donc, il y avait ce problème et avec le $3^{\text {ème }}$ PROSIT, le tout retombait sur moi. (M7)

Oui, globalement, j'ai apprécié la dynamique d'équipe. Cependant, il faut dire qu'il y avait l'absentéisme et parfois certains avaient tendance à nous imposer leurs idées 
même à tord. Mais, on peut mettre çà au compte du manque d'expériences des uns et des autres. En dépit de tout, un consensus s'est dégagé. (B7)

\section{Le rapport social à l'environnement}

27 Certains participants font état du rapport social à l'environnement :

L'APP pourrait favoriser le développement de professionnels capables de susciter et d'accompagner un bouleversement des comportements chez les populations notamment en milieu rural, mais cela vaudra vraiment le coût. À mon avis, cela devrait amener les gens à porter un regard critique sur les phénomènes. Par exemple, l'hygiène peut être un moyen de lutte contre la pauvreté. (R5)

La prise en compte de ces considérations est importante si l'on veut mieux comprendre la dynamique des rapports sociaux à l'environnement dans le but de planifier des situations de formation appropriées, d'autant plus que notre recherche s'inscrit dans une perspective réflexive en vue de contribuer à la transformation des pratiques sociales. L'importance que les participants attachent à l'amélioration des conditions de vie des populations est illustrée par le commentaire récurrent suivant : "La démarche devrait contribuer à l'amélioration des conditions de vie des populations.» (N6;S7; C1). Les commentaires ci-dessus émergent des discours des étudiants et témoignent des controverses lors de la construction de leur propre argumentaire mais aussi de la déconstruction de celui des autres, non seulement sur le plan des savoirs mais aussi sur celui des valeurs.

\section{Que retenir des enjeux socio-politiques liés à la mise en œuvre de I'APP ?}

29 D'une part, il ressort des travaux de Barry (2001) que les populations guinéennes, essentiellement agricoles et pour la plupart analphabètes, portent préjudice à l'environnement à travers certaines de leurs pratiques coutumières courantes. D'autre part, l'analyse des résultats de la mise en œuvre de l'APP montre qu'en Guinée, la culture de l'enseignement et de l'apprentissage est caractérisée par l'individualisme et la compétition. Comme mentionné dans l'exposé de la problématique, l'une des limites de l'enseignement supérieur guinéen provient du fait que dans les pratiques en classe, on applique souvent une prescription venue d'en haut sans nécessairement l'appuyer et l'adapter avec des exemples tirés de la vie quotidienne de celui qui apprend. Les propos de certains participants corroborent ce constat. Bon nombre d'entre eux affirment être obligés de composer avec des savoirs qui prennent la forme de théories figées, voire de vérités révélées.

En s'appuyant sur la problématique de cette recherche, deux phénomènes sont reliés à cette situation : 1) les enseignants occupent une position d'autorité face aux étudiants ; 2) le rôle de l'étudiant consiste à recevoir l'information de manière passive, à reproduire et à mémoriser celle-ci et à la restituer sans aucune forme de critique. Rappelons ici que la tradition éducative (dominée par la logique transmissive) dans laquelle ces enseignants ont évolué rend le défi encore plus exigeant. Comme le font observer Ouellet et Guilbert (1997), l'enseignant devrait laisser de côté son statut d'autorité et d'expert pour guider, faciliter l'apprentissage et introduire un espace de débat en classe. Pour renforcer cette idée, Bader et Sauvé (2011) soulignent la pertinence de situations éducatives particulières faisant appel aux débats. 
31 Pour sa part, Sauvé (1997a, p. 47) souligne que «La prévention et la résolution des problèmes environnementaux, de même que la gestion des ressources, font l'objet de controverses entre divers protagonistes dont les croyances, les attitudes et les valeurs different ». Dans la même logique, Albe (2009, C4) faisait remarquer que pour enseigner des controverses socio-scientifiques,

L'enseignant ne peut s'appuyer sur des savoirs stabilisés dans une communauté socialement reconnue comme productrice de savoirs mais se trouve au contraire face à une diversité de groupes sociaux divisés, chacun d'entre eux produisant des savoirs et des arguments, également marqués par une grande diversité. Dans un tel contexte, le manuel d'enseignement ne propose que peu d'aide pour l'enseignement des controverses scientifiques soulevant également des controverses en société et comportant une dimension sociale, politique, économique, éthique, etc.

En outre, compte-tenu de la tradition éducative guinéenne, l'apport actif des participants dans la co-construction de l'apprentissage constitue une difficulté majeure : produire un écrit réflexif et critique. En dépit de moments de confrontation apparente, des discussions intenses se sont avérées très fécondes dans le processus de formation. À nos yeux, et tel que suggéré par Orellana (2002, p. 328), l'intégration de la pratique de la métacognition individuelle et collective en cours d'apprentissage devrait constituer un véritable apport au processus de co-construction des savoirs, à sa dynamique de mise en œuvre et au développement personnel et professionnel des participants.

\section{En guise conclusion}

Cette étude a contribué à mieux cerner les enjeux sociopolitiques dans la mise en œuvre de l'apprentissage par problèmes au regard du développement de compétences associées au champ de l'éducation relative à l'environnement chez les étudiants du CERE. Toutefois, elle n'épuise pas toutes les questions ayant trait aux enjeux associés à la mise en œuvre de l'APP.

Dans le processus global de mise en oeuvre de l'APP, deux enjeux socio-politiques majeurs, tels que perçus par les participants, semblent se dégager. Premièrement, les défis de l'adoption d'une dynamique participative. Deuxièmement, la prise en compte du rapport social à l'environnement. Maintenir les échanges et les interactions entre les étudiants mais aussi avec le tuteur, ainsi que le dialogue et la participation aux débats, ont été des enjeux majeurs dans la mise en œuvre de l'APP. Des controverses importantes associées à l'innovation pédagogique ont été évoquées en ce sens. Les travaux de Robottom et Hart (1993, p. 24) s'inscrivent dans la même perspective: " l'éducation relative à l'environnement de type critique doit aller au-delà des investigations des étudiants concernant les systèmes de valeurs à la base des questions environnementales ».

En termes de prospectives, quelques avenues se dessinent. Il serait intéressant de mener à plus long terme une étude de cas évaluative (multi-cas) du transfert des apprentissages en contexte d'intervention inspirée de l'APP. À ce titre, chaque étudiant ayant déjà vécue cette expérience pourrait être considéré comme un cas afin d'évaluer l'impact de l'APP au regard du développement de ses compétences associées au champ de l'ERE, en examinant le transfert dans sa propre pratique professionnelle. 


\section{BIBLIOGRAPHIE}

Albanese, M. A. et Mitchell, S. (1993). Problem-based learning : review of literature on its outcome and implementation issues. Academic Medecine. 68(1), 52-78.

Albe, V. (2009). Enseigner des controverses. Rennes : Presses Universitaires de Rennes.

Bader, B., Sauvé, L. (2011). Éducation, environnement et développement durable. Vers une écocitoyenneté critique. Québec : Presses de l'Université Laval, collection « l'espace public».

Bah, M. B. (2012). Apports, limites et enjeux de l'apprentissage par problèmes pour le développement de compétences associées au champ de l'éducation relative à l'environnement : une étude de cas en milieu universitaire. (Thèse de doctorat inédite, Université de Québec à Montréal, Québec, Canada).

Banque mondiale (2003). Rapport de stratégie d'appui au pays. Pour la République de Guinée. 10 juin 2003. Numéro 25925 GUI, Bureau des opérations pour la Guinée, Département-pays 11, Région Afrique.

Barry, A. (1998). L'apport des parents à la définition de nouvelles fonctions pour l'enseignement primaire en République de Guinée. (Thèse de doctorat inédite, Université du Québec à Montréal, Canada).

Barry, L. (2001). École propre-École verte: Une voie pour qualifier l'école, mobiliser la communauté et protéger l'environnement - une expérience guinéenne à partager. Monographies innodata - 10, UNESCO, BIE, 2001.

Berthelot, M. (2007). Étude de la contribution du programme de formation information pour l'environnement à la pérennisation de l'éducation relative à l'environnement dans l'enseignement primaire sénégalais. (Thèse de doctorat inédite, Université Laval, Québec, Canada).

Bertrand, Y. (1998). Théories contemporaines de l'éducation, 4 è édition. Montréal, Québec : Éditions Nouvelles, Chronique sociale, Lyon, France.

Boud, D. et Felleti, G. (1991). (dir.). The Challenge of Problem-Based Learning. New York : St-Martin's Press.

Bridges, E. M. (1992). Problem-based learning for administrators. Eric clearinghouse on education management. Oregon : University of Oregon.

Brown, J. S., Collins, A. et Duguid, P. (1989). Situated cognition and the culture of learning. Educational Researcher, 18(1), 32-42.

Commission Européenne Résidente en Guinée. (2007). Profil environnemental de la Guinée. Document de travail de base, Conakry, Guinée.

Dalceggio, P. (1991). Qu'est-ce qu'apprendre? Montréal : Service d'aide à l'enseignement, Université de Montréal.

Dubeau, A. (2001). L'apprentissage par problème. Trait d'Union. Université de Sherbrooke, Québec, $4(4), 2-3$

Greeno, J. G. (1988a). A perspective on thinking. IRL Report 88-0010. Palo Alto : Institute for Research on Learning.

Greeno, J. G. (1988b). Situations, mental models and generative knowledge. IRL Report 88-0005. Palo Alto : Institute for Research on Learning. 
Jorro, A (2006). Devenir ami critique. Avec quelles compétences et quels gestes professionnels? Mesure et évaluation en éducation, 29(1), 31-44

Kaufman, A., Obenshain, S. S. (1985). Origins. Dans A. Kaufman (dir.), Implementing problem-based medical education. Lessons from successful innovations (1-15). New York : Springer publishing company.

Lave, J. (1988). The culture of acquisition and the practice of understanding. IRL Report 88-0087. Palo Alto : Institute for Research on Learning.

L'Écuyer, R. (1990). Méthodologie de l'analyse développementale du contenu. Méthode GPS et concept de soi. Québec: Presses de l'Université du Québec.

Margetson, D. (1991). Is there a Future for Problem-Based Education? Higher Education Review. 23(2), 33-47. New York : Saint-Martin's Press.

Orellana, I. (2002). La communauté d'apprentissage en éducation relative à l'environnement : signification, dynamiques et enjeux. Thèse de doctorat en éducation inédite, Université du Québec à Montréal, Canada. Récupérée sur www.archipel.uqam.ca/7213/

Ouellet, L. et Guilbert, L. (1997). L'opérationnalisation d'un modèle socio-constructiviste d'apprentissage par problèmes en milieu collégial. Éducation et francophonie, XXV(1). Récupéré le 19 septembre 2008 sur : http://www.acelf.ca/c/revue/pdf/EF-25-1-130_OUELLET.pdf

Paillé, P., Mucchielli, A. (2012). L'analyse qualitative en sciences humaines et sociales. Paris : Armand Colin, collection U, 3è édition.

Robottom, I., Hart, P. (1993). Research in environmental education. Engaging the debate. Geelong, Australie : Deakin University Press.

Saint-Jean, M. (1994). L'apprentissage par problèmes dans l'enseignement supérieur. Service d'aide à l'enseignement, Université de Montréal, Québec.

Sauvé, L. (1997a). Pour une éducation relative à l'environnement. Éléments de design pédagogique Guide de développement professionnel à l'intention des éducateurs. Montréal : Guérin, 2e édition.

Sauvé, L., Orellana, I. (2008). Éditorial : Conjuguer rigueur, équité, créativité et amour : L'exigence de la criticité en éducation relative à l'environnement. Éducation relative à l'environnement : Regards Recherches Réflexions, 7, 7-20.

Sauvé, L. (2009). Pourquoi développer l'éducation relative à l'environnement aujourd'hui ? Assises nationales de l'éducation à l'environnement vers un développement durable, Caen, France, 28 octobre 2009. Contribution par vidéos en plénière.

Tardif, J. (1997). Pour un enseignement stratégique : l'apport de la psychologie cognitive. Montréal : Les Éditions Logiques.

Walton, H. J. et Matthews, M. B. (1989). Essentials of problem-based learning. Medical Education, 23, 542-558.

\section{NOTES}

1. La moyenne d'âge des étudiants est de 33 ans avec une concentration entre 30 et 35 ans (environ $50 \%$ des étudiants). La répartition des genres est asymétrique (environ $75 \%$ d'hommes et $25 \%$ de femmes). Dans cette recherche, le corpus est issu à la fois i) d'échanges oraux (les propos des étudiants tenus en entrevue, les difficultés rencontrées par les participants), ii) des écrits (les notes d'observation du chercheur et de son ami critique ; les réponses consignées par 
chaque étudiant lors de son travail individuel) et iii) de traces audio-visuelles (les séquences vidéo issues des moments d'observation des travaux d'équipe et des plénières).

\section{RÉSUMÉS}

Cet article traite des enjeux socio-politiques, tels que perçus par vingt et un étudiants du programme de Master en sciences de l'environnement du Centre d'étude et de recherche en environnement (CERE) de l'Université Gamal Abdel Nasser de Conakry, durant la mise en œuvre de l'apprentissage par problèmes (APP) dans le cadre d'un cours d'éducation relative à l'environnement (ERE). Échelonnée sur cinq mois (de janvier à mai 2011), cette mise en œuvre visait le développement de compétences associées au champ de l'ERE chez ces étudiants. Les enjeux socio-politiques de la dynamique de mise en œuvre de l'APP sont présentés et discutés afin d'en dégager des avenues à explorer pour le développement de compétences associées au champ de l'ERE chez ces étudiants.

This article deals with socio-political issues, as perceived by twenty-one students of the Master's program in Environmental Sciences of Centre d'étude et de recherche en environnement (CERE) of Gamal Abdel Nasser University of Conakry, during the implementation of problem-based learning (PBL) as part of an environmental education (EE) course. Spread over five months (from January to May 2011), this implementation aimed at developing skills associated with the field of EE for these students. The socio-political issues of the dynamics of implementation of PBL are presented and discussed in order to identify avenues to explore for the development of skills associated with the field of EE for these students.

\section{INDEX}

Index géographique : République de Guinée

Mots-clés : apprentissage par problèmes, enjeux socio-politiques, apprentissage contextualisé

Keywords : problem-based learning, socio-political issues, contextualized learning

\section{AUTEUR}

\section{MAMADOU BHOYE BAH}

Mamadou Bhoye Bah est titulaire d'un doctorat (Ph.D.) en éducation et d'une Attestation d'éducation relative à l'environnement (programme court) de l'Université du Québec à Montréal. Il est également titulaire d'un diplôme d'études approfondies (DEA) en sciences de l'environnement du Centre d'étude et de recherche en environnement (CERE) de l'Université Gamal Abdel Nasser de Conakry. Enseignant-chercheur (Maître de Conférences) au même Centre, il dispense le cours d'éducation relative à l'environnement (ERE) et co-enseigne le cours de méthodologie de la recherche au programme de Master en sciences de l'environnement. Ses activités de formation et de recherche s'inscrivent dans le mouvement du renouveau pédagogique universitaire qu'il explore notamment par la dynamique de la mise en œuvre de 
l'apprentissage par problèmes (APP) au regard du développement de compétences associées au champ de l'éducation relative à l'environnement. 